\title{
A new histochemical technique of use in the interpretation and diagnosis of adenocarcinoma and villous lesions in the large intestine ${ }^{1}$
}

\author{
C. F. A. CUlling, P. E. REID, ANN J. WORTH, AND W. L. DUNN
}

From the Department of Pathology, University of British Columbia, The Cancer Control Agency of BC, and Vancouver General Hospital, Vancouver, BC, Canada

SUMMARY The periodic acid-thionin Schiff/potassium hydroxide/periodic acid-Schiff (PAT/KOH/ PAS) procedure has been used to investigate the histochemical staining characteristics of the mucins found in adenocarcinoma and villous lesions of the large intestine. The 46 blocks examined represented 58 lesions from 37 patients, all of whom had had resections for carcinoma of the colon.

In sharp contrast to normal colon, none of the adenocarcinomas stained red with the PAT/KOH/ PAS. With two exceptions the poorly and moderately differentiated adenocarcinomas stained blue, whereas of the well differentiated lesions half were blue and half purple. The malignant villous lesions demonstrated the same trends, although a larger percentage were purple. None of the benign lesions stained blue.

It is suggested that malignancy in the colon is accompanied by an increase in blue staining in the $\mathrm{PAT} / \mathrm{KOH} / \mathrm{PAS}$ technique and that such staining may be of value in the interpretation of highly atypical adenoma where it might identify the onset of malignancy. This change in staining indicates a distinct alteration in the chemistry of the mucins which we interpret as a reduction in the degree of side chain $\mathrm{O}$-acylation of their constituent sialic acids.

We have shown (Culling et al., 1971, 1974a, 1974b, 1976; Reid et al., 1973, 1974, 1975a, 1975b, 1976) that both chemical and histochemical procedures can be used to demonstrate the types and location of a variety of $\mathrm{O}$-acylated sialic acids, and that in the normal colon the latter are reasonably consistent in nature. This fact was utilised in a previous study (Culling et al., 1975) to differentiate those mucinproducing metastases arising from adenocarcinoma of the lower gastrointestinal tract from those arising elsewhere. At that time we noted that $30 \%$ of such primary tumours were $\mathrm{KOH} / \mathrm{PAS}$ negative (Culling et al., 1975) and that this might prove to be of clinical significance. It was surmised that this latter type of tumour could have arisen from a different cell type or that a greater degree of dysdifferentiation had occurred. Further, it was thought possible that malignant changes could produce an alteration in the structure of the $\mathrm{O}$-acylated sialic acids of the epithelial mucins produced.

${ }^{1}$ Supported by the Medical Research Council of Canada (MA 4375)

Received for publication 31 March 1977
Recently we have devised a new method for demonstrating both $\mathrm{KOH} / \mathrm{PAS}$ positive (Culling et al., 1971, 1974a; Reid et al., 1973) and KOH/PAS negative mucins in contrasting colours in the same section (Culling et al., 1976). The present paper describes the results of the application of this technique to a number of adenocarcinomas and polyps of the large intestine.

\section{Material}

The specimens investigated were obtained from the surgical pathology department of the Vancouver General Hospital and the Cancer Control Agency of British Columbia. The 46 blocks examined (containing 58 lesions) were from 37 patients, all of whom had had a resection for carcinoma of the colon. The lesions were selected to provide a representative sampling of all areas of the large bowel from caecum to rectum, and covered a wide range of diagnoses including benign adenomatous polyp, villous adenoma with varying degrees of atypia and malignancy, and deeply invasive carcinoma. There was some bias in favour of those specimens in which 
there were multiple lesions, as these provided an opportunity to examine variability in interlesional staining in the same patients. For the same reason all the blocks contained a cuff of histologically normal colonic mucosa for comparison with the lesion. The tissues had been fixed in formalin, routinely processed, and embedded in Paraplast and sections were cut at 5 microns.

\section{Histochemical techniques}

All standard techniques were performed as described by Culling (1974):

1 Periodic acid-Schiff (PAS) to demonstrate periodate reactive vicinal diols (1:2 glycol groups) in red;

2 Periodate-borohydride technique (PBT) to render periodate reactive vicinal diols non-reactive to PAS (Reid et al., 1973);

3 Saponification $(\mathrm{KOH})$ with $0.5 \%$ potassium hydroxide in $70 \%$ ethanol for $30 \mathrm{~min}$ at room temperature to deacetylate sialic acid residues;

$4 \mathrm{PBT} / \mathrm{KOH} / \mathrm{PAS}$ to demonstrate $\mathrm{KOH} / \mathrm{PAS}$ positive structure (Reid et al., 1973; Culling, 1974) in isolation.

5 Periodic acid-thionin Schiff (PAT) (Culling et al., 1976) to demonstrate periodate reactive vicinal diols in blue;

$6 \mathrm{PAT} / \mathrm{KOH} / \mathrm{PAS}$ (technique given below) to demonstrate normally PAS reactive material in blue, while $\mathrm{KOH} / \mathrm{PAS}$ reactive tissue components (Oacylated sialic acids) show as red, mixtures being purple (Culling et al., 1976).

\section{PAT/KOH/PAS TECHNIQUE}

Routine formol-calcium fixed, Paraplast embedded tissue sections were used in this study, but cryostat or Paraplast embedded sections fixed in any manner that does not interfere with or remove vicinal diols may be used equally well. Sections must be floated onto clean, grease-free slides. This will avoid the loss of sections during staining. Albumen or chrome alum gelatin (Culling, 1974) may be used if sections become detached, but we do not find this necessary.

\section{THIONIN SCHIFF REAGENT}

One gram of thionin was dissolved in $100 \mathrm{ml}$ of distilled water with the aid of heat. The solution was then cooled, $0.75 \%$ thionyl chloride added, and left overnight. Activated charcoal $2.0 \%$ was added, and the flask was shaken and immediately filtered. It was then stored in a stoppered dark bottle in the refrigerator (where it will keep for three to four weeks).

\section{Method}

1 Bring slides to water.
2 Place in $1 \%$ aqueous periodic acid (freshly prepared) for 30 minutes.

3 Wash in running tap water for 10 minutes.

4 Place in thionin Schiff reagent for 30 minutes.

5 Wash in running tap water for 10 minutes.

6 Rinse in $70 \%$ ethanol, and treat with $0.5 \%$ potassium hydroxide in $70 \%$ ethanol for 30 minutes.

7 Wash gently in tap water; avoid dislodging section.

8 Place in fresh $1 \%$ periodic acid for 10 minutes.

9 Wash gently in running tap water for 10 minutes.

10 Place in standard Schiff reagent for 30 minutes.

11 Wash gently in running tap water for 10 minutes.

12 Dehydrate, clear, and mount in HSR (or other resin mountant).

Results: PAS positive material will be blue, and $\mathrm{KOH} / \mathrm{PAS}$ positive materials will be red, mixtures being purple.

\section{Results}

Sections were examined and graded morphologically by one of us (AW) using routinely stained haematoxylin and eosin slides. Further sections were then stained by the histochemical techniques described above and, without the morphological grading being available, were graded on the basis of the type (colour) of mucin produced. Mucin was classified by the PAT/KOH/PAS method as being blue, purple, or red. It was realised that this was subjective, and our original classification was blue, blue-purple, purple, red-purple, and red and could easily have been further subdivided; however, it was finally felt that the colours could most easily be divided into predominantly red or blue with the single class of purple for intermediates. It should be remembered that normal colon gives a reasonably clear red by this method (Fig. 1). The PBT/KOH/PAS treated slides were graded as either weakly positive $( \pm)$ when there was even a slight red colour present, as positive $(+)$ when there was a definite to strong. red colour, or negative $(-)$ when there was no red colour present. PBT/PAS controls must be included with every batch of slides stained and these controls must not contain any red colour.

After the slides had been read on a histochemical basis alone, the classification was obtained and Tables 1 and 2 were constructed. It should be noted that several hundred slides of normal colon have been examined which were all strongly positive by the PBT/KOH/PAS and stained red by the PAT/KOH/PAS technique.

Tables 1 and 2 show that, in sharp contrast to the normal colon, none of the malignancies stained red with the PAT/KOH/PAS method, and half were 

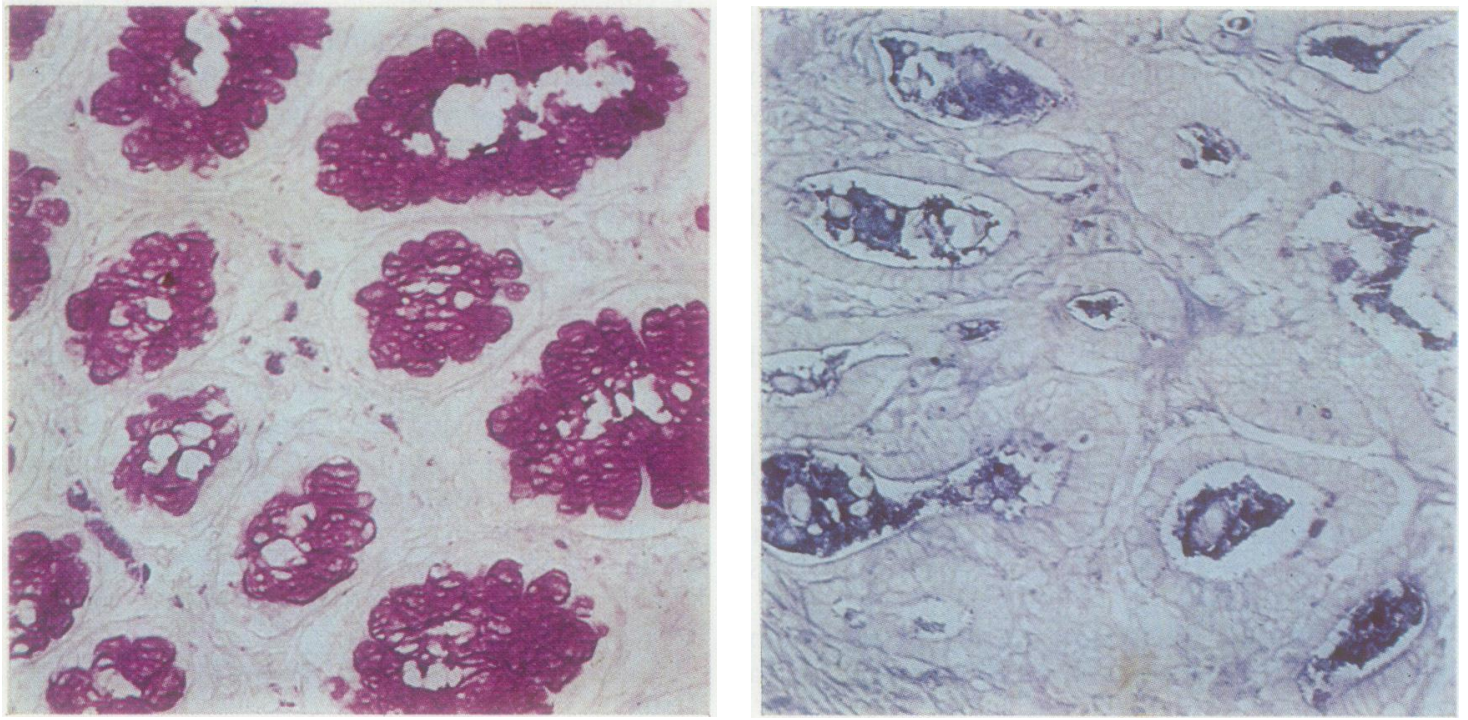

2
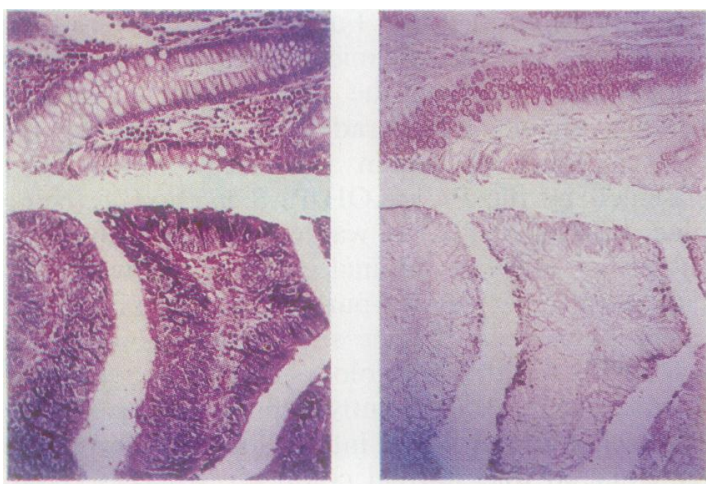

$3 a$

$3 \mathrm{~b}$

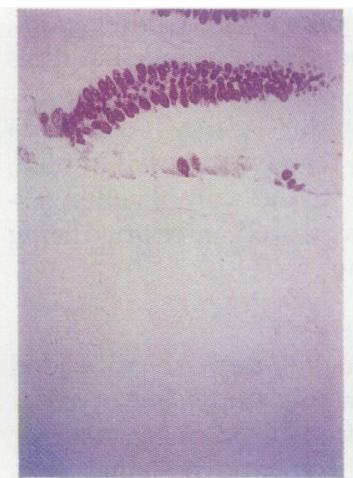

$3 c$

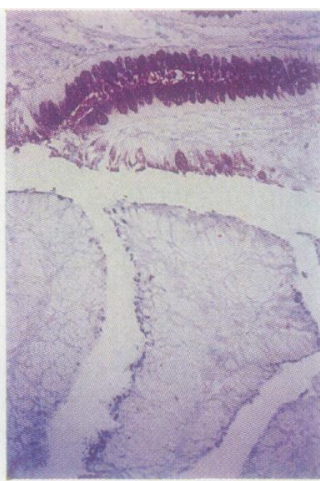

$3 d$

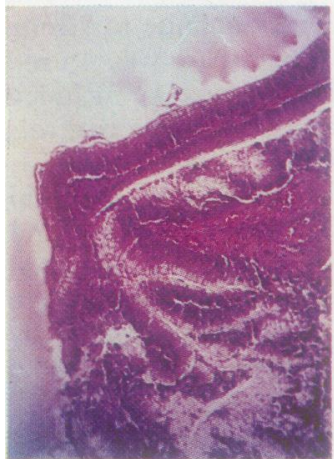

$4 a$

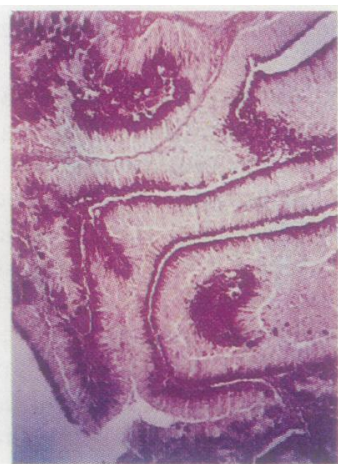

$4 b$

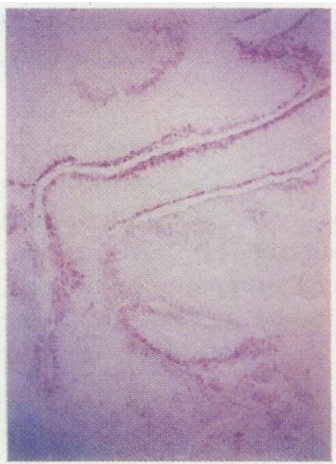

$4 c$

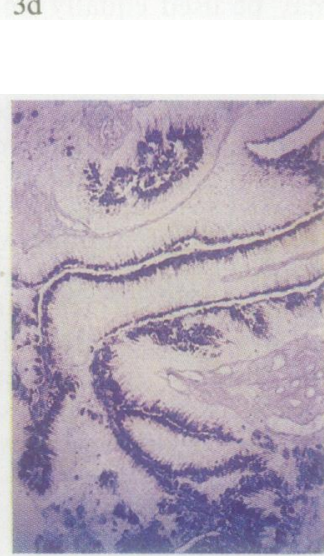

$4 d$ 
Fig. 1 Normal colon stained by $P A T / K O H / P A S$ showing normal reddish colour of the epithelial mucin.

Fig. 2 Adenocarcinoma of colon, stained by PAT/KOH/ $P A S$, the epithelial mucin of which, in contrast to that in

Fig. 1, clearly shows blue staining.

Fig. 3 Sections of a well-differentiated adenocarcinoma, which shows a sharp transition from histologically normal to malignancy: (a) Haematoxylin and eosin; (b) Routine $P A S$ stain. Note increased intensity of staining of tumour mucin; (c) PBT/KOH/PAS. Note strong staining of normal mucin contrasting with its absence in tumour,

(d) PAT/KOH/PAS. Note correspondence between PAS staining (Fig. $3 b$ ) and blue staining in the tumour mucin and difference in colour between normal and malignant tissue mucin.

Fig. 4 Sections of a villous adenoma with a histologically 'borderline' in-situ carcinoma, the epithelial mucin of which appears to contain $\mathrm{C}_{7} \mathrm{O}$-acylated sialic acid: (a) Haematoxylin and eosin; (b) Routine PAS. Note intensity of $P A S$ staining and compare with normal mucin in Fig. 3b; (c) PBT/KOH/PAS. Note all mucin stains, but less strongly than in the normal in Fig. $3 c$;

(d)PAT/KOH/PAS. Note strong blue staining of all mucin. The presence of clear blue staining, along with a positive $P B T / K O H / P A S$ (Fig. $4 c$ ), indicates the presence of $C_{7}$ $O$-acylated sialic acid (see Table 3).
PBT/KOH/PAS negative. With two exceptions the poorly and moderately differentiated adenocarcinomatous areas stained blue (Fig. 2). In the well differentiated lesions half were blue and half were various hues of purple. The PBT/KOH/PAS staining reactions were more variable; only two of the less well differentiated lesions were distinctly positive. Ten of the 24 blocks contained a few scattered positively staining granules. The well differentiated cases showed a wider range of reactions.

The malignant villous lesions demonstrated the same trends although a larger percentage were purple with the PAT/KOH/PAS method and the majority displayed some PBT/KOH/PAS positivity.

All the benign lesions were PBT/KOH/PAS positive and none was blue with PAT/KOH/PAS. Only one block (27C), in which there was no histologic evidence of malignancy, contained blue. This block was from an apparently benign area associated with a malignant villous lesion immediately adjacent to the carcinomatous focus. None of the other benign lesions studied was found to have any malignant foci within.

The staining patterns varied between lesions and

Table 1 Staining characteristics of adenocarcinomas of colon

\begin{tabular}{|c|c|c|c|c|c|c|c|c|}
\hline \multicolumn{2}{|c|}{ Classification } & \multirow[t]{2}{*}{ Case No. } & \multicolumn{3}{|c|}{$P A T / K O H / P A S$} & \multicolumn{3}{|c|}{$P B T / K O H / P A S$} \\
\hline & & & Blue & Purple & Red & + & \pm & $\mathbf{0}$ \\
\hline \multicolumn{2}{|c|}{ Poorly differentiated } & 1 & $x$ & & & & & $x$ \\
\hline$"$ & " & 2 & $x$ & & & & $x$ & \\
\hline ", & " & 3 & $x$ & & & & $x$ & \\
\hline , & ", & 4 & $x$ & & & & $x$ & \\
\hline , & $"$ & 5 & $x$ & & & & & $x$ \\
\hline ", & $"$ & 6 & $x$ & & & & & $x$ \\
\hline ", & $"$ & 7 & $x$ & & & & & $x$ \\
\hline ", & $"$ & 8 & $x$ & & & & & $x$ \\
\hline ", & ", & 9 & $x$ & & & & $x$ & \\
\hline$"$ & $"$ & 10 & $x$ & & & & $x$ & \\
\hline ", & " & 11 & $x$ & & & & & $x$ \\
\hline$"$ & ," & 12 & $x$ & & & & & $x$ \\
\hline$"$ & $"$ & 13 & & $x$ & & $x$ & & \\
\hline 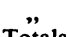 & " & 14 & $x$ & & & & $x$ & \\
\hline Totals & & 14 & 13 & 1 & & 1 & 6 & 7 \\
\hline \multicolumn{2}{|c|}{ Moderately differentiated } & 15 & $x$ & & & & & $x$ \\
\hline$"$ & $"$ & 16 & $x$ & & & & $x$ & \\
\hline ", & , & 17 & $x$ & & & & & $x$ \\
\hline ", & ", & 18 & & $x$ & & $x$ & & \\
\hline „, & ", & 19 & $x$ & & & & & $x$ \\
\hline ", & $"$ & $20 \mathrm{a}$ & $x$ & & & & & $x$ \\
\hline ", & , & $20 \mathrm{~b}$ & $x$ & & & & $x$ & \\
\hline$"$ & $"$ & $21 \mathbf{a}$ & $x$ & & & & & $x$ \\
\hline$"$ & ," & $21 \mathrm{~b}$ & $x$ & & & & & $x$ \\
\hline ", & ", & 22 & $x$ & & & & $x$ & \\
\hline Totals & $"$ & 10 & 9 & 1 & & 1 & $\hat{3}$ & 6 \\
\hline \multicolumn{2}{|c|}{ Well differentiated } & $23 \mathbf{a}$ & & $x$ & & $x$ & & \\
\hline$"$ & " & $23 b$ & & $x$ & & $x$ & & \\
\hline & $"$ & 24 & $x$ & & & & & $x$ \\
\hline$"$ & " & 25 & & $x$ & & & $x$ & \\
\hline & " & $26 a$ & $x$ & & & & & $x$ \\
\hline ” & $"$ & $26 \mathrm{~b}$ & $x$ & & & & $x$ & \\
\hline$"$ & " & $27 a$ & & $x$ & & & $x$ & \\
\hline Totals & & 7 & 3 & 4 & & 2 & 3 & 2 \\
\hline
\end{tabular}


Table 2 Staining characteristics of adenomatous lesions

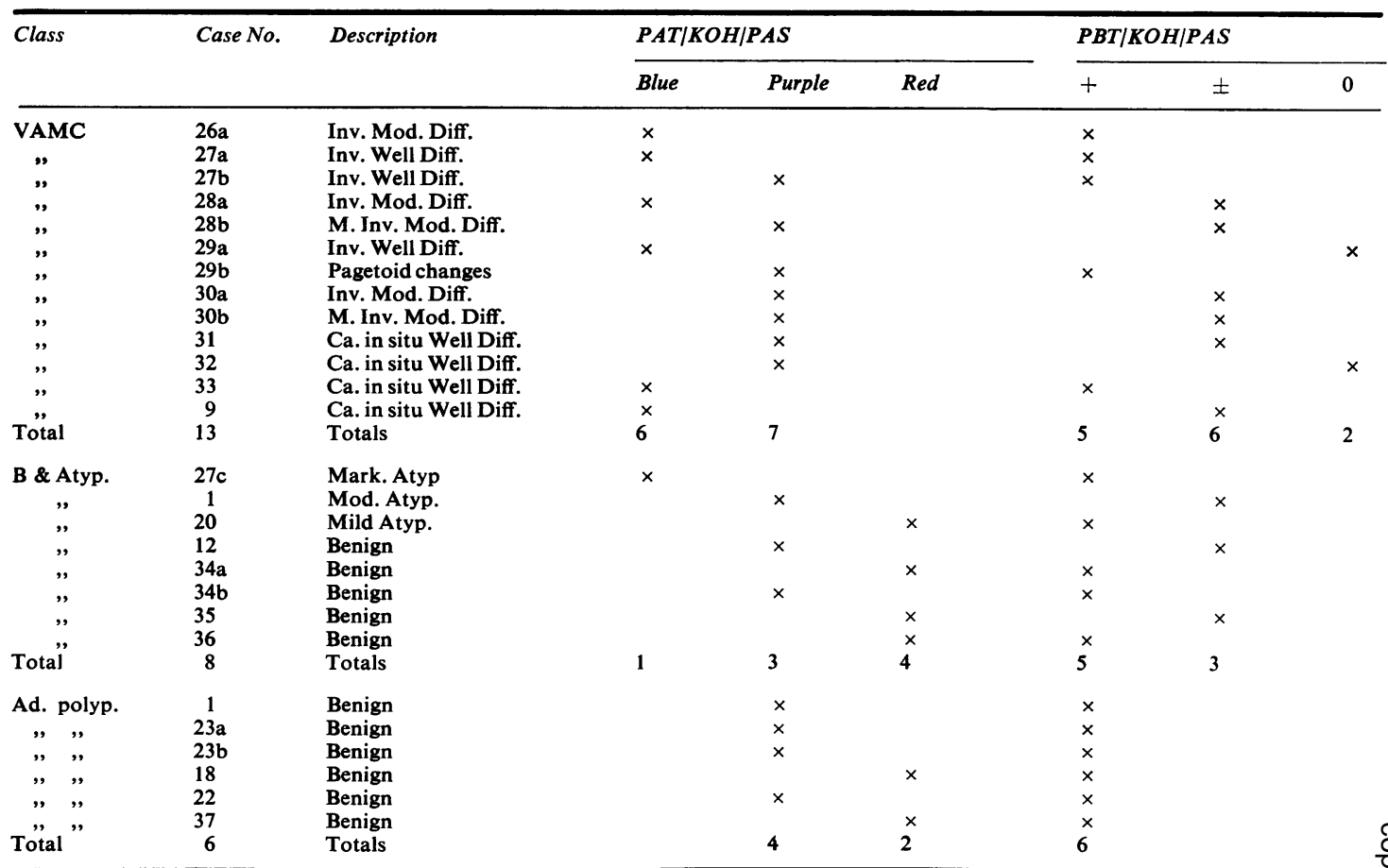

VAMC = villous adenoma-malignant changes; $B$ \& Atyp. = benign and atypical; Ad. polyp. = adenomatous polyp; Inv. = invasive ;

Mod. = moderate; Mark. = markedly; Atyp. = atypical.

within the same lesion. Many of the carcinomas, particularly the poorly differentiated lesions, showed little staining by either method. In some cases pools of blue mucoid material were present within the malignant acini while in others a thin rim of blue etched the luminal aspect of the cells. With light microscopy it was not possible to determine whether this represented an alteration in the glycocalyceal portion of the cells or represented adherent luminal secretions. We hope to elucidate this latter problem with some new electron microscope histochemical methods now being developed. In some instances a beaded row of small blue droplets was seen in the luminal aspect of the cytoplasm. These droplets tended to be smaller than the red staining droplets seen in the cytoplasm of the adjacent normal mucosa or within the occasional small residual island of normal epithelium incorporated within the carcinoma. Some carcinomas contained more widely dispersed and randomly distributed intracytoplasmic droplets of varying hues of blue and purple. In one interesting case (29B), in which intraepithelial pagetoid changes were associated with a large underlying anorectal adenocarcinoma of predomin- antly blue reaction, the intraepithelial component contained many vacuoles ranging from reddish purple to blue. Only very rarely were normally staining red droplets seen in the malignant cells.

The transition between the normal (or benign) and malignant mucosa was occasionally very striking with an abrupt change in the staining reaction. In other cases a more gradual transition was present which corresponded to the transition seen in the $\mathrm{H}$ and $\mathrm{E}$ sections from normal through atypical to malignant epithelium. In eight cases the apparently normal adjacent epithelium presented a reddishpurple tone rather than the red of the normal colonic epithelium in the previously studied control cases (Fig. 3).

Since the KOH/PAS effect (Culling et al., 1971, 1974a, 1976; Reid et al., 1973) is responsible for the red component of the PAT/KOH/PAS staining, it will be seen that the results shown in Tables 1 and 2 for the PBT/KOH/PAS are consistent with those obtained with the PAT/KOH/PAS with three exceptions. In these three cases the mucins stained blue with PAT/KOH/PAS and a definite red by the PBT/KOH/PAS (Fig. 4). In such KOH/PAS positive mucins the sialic acid present will probably be 
predominantly $\mathrm{C}_{7}$ O-acylated (Culling et al., 1976).

It should be remembered that the blue component of the PAT/KOH/PAS staining represents normal PAS reactivity and must not be confused with the action of thionin as a basic dye (Culling et al., 1976). These results, therefore, suggested that the standard PAS reactivity of colonic mucin is frequently increased in adenocarcinomas, by comparison with normal colon, and a retrospective study confirmed this somewhat surprising finding.

\section{Discussion}

We have shown that an examination of sections stained by the PAT/KOH/PAS and the PBT/KOH/ PAS enables us to determine the $\mathrm{O}$-acyl substituent patterns of the polyhydroxy side chains of the sialic acids of normal colonic epithelial mucins (Culling et al., 1974a, 1976). Red staining in the PBT/KOH/ PAS implies substitution at positions $\mathrm{C}_{7}$ and/or $\mathrm{C}_{8}$, or any other pattern of side chain di- or tri-substitution; red staining in the PAT/KOH/PAS identifies only those sialic acids substituted at $\mathrm{C}_{8}$ (or di- or tri-substituted). Blue staining in the latter is due to sialic acids substituted at either $C_{7}$ or $C_{9}$ or which are unsubstituted (normally PAS reactive); purple staining will obviously indicate a mixture. This scheme is illustrated in Table 3 (taken from our previous paper-Culling et al., 1976). As will be seen in Tables 1 and 2, three cases produced mucin which stained blue by the PAT/KOH/PAS and red by the PBT/KOH/PAS (Fig. 4). Based upon our previous studies (Table 3 ), the $\mathrm{O}$-acylated sialic acids of these mucins are predominantly substituted at position $\mathrm{C}_{7}$.

As a result of this study it appears that the onset of malignancy in the colon is accompanied by an increase in blue staining. In histochemical terms this indicates a distinct change in the chemistry of the mucins being produced. We assume that this indicates a reduction in the degree of $\mathrm{O}$-acylation of the sialic acids, although it is possible that other sites with potential vicinal diols might be involved. Such a change in structure could be due to the deletion, or alteration in the properties, of an O-acylating enzyme or enzymes. In those cases where the mucin

Table 3 Histochemical identification of side chain O-acylated sialic acids (Culling et al., 1976)

\begin{tabular}{lll}
\hline Side chain & $P A T / K O H / P A S$ & $P B T / K O H / P A S$ \\
\hline $\mathrm{C}_{0}$ & Blue & Unstained \\
$\mathrm{C}_{7}$ & Blue & Red \\
$\mathrm{C}_{8}$ & Blue & Unstained \\
$\mathrm{C}_{8}, \mathrm{C}_{7,8}$ & Red & Red \\
$\mathrm{C}_{7,8} ; \mathrm{C}_{7,8,8}$ & Red & Red \\
\hline
\end{tabular}

is predominantly $\mathrm{C}_{7}$, the change appears to be quite specific. In four of the sections there was a sharp morphological transition from normal to malignant which was accompanied by a similar sharp change in histochemical staining. In the majority of sections this histochemical change was less obvious, and in a few sections the apparently normal epithelial mucin farthest from the tumour edge was a distinct purple.

Filipe and others (Filipe, 1969, 1972; Filipe and Branfoot, 1974; Dawson and Filipe, 1976a, 1976b) have recorded histochemical changes in the transitional zone between normal and tumour tissue. These consisted of 'an increase of sialomucins accompanied usually by a decrease or absence of the sulphomucins which are predominant in the normal mucosa of the large intestine' (Filipe and Branfoot, 1974; Dawson and Filipe, 1976b). In addition, they noted (Dawson and Filipe, 1976b) that in the transitional mucosa, adjacent to carcinoma, a far more positive reaction is observed' as compared with normal when using the periodic acid-chromic acid-silver methenamine method for glycoproteins (silver PAS method). Furthermore, Filipe and Cooke (1974), in a biochemical study, reported that transitional mucosa showed, upon paper chromatography following hydrolysis, two thiobarbituric acid positive components. The faster of these was $\mathrm{N}$-acetyl-neuraminic acid and the slower was probably an $\mathrm{O}$-acetylated $\mathrm{N}$-acetyl neuraminic acid. Normal mucosa gave only a single component with the mobility similar to that of the slower component of transitional mucosa. The above data appear directly to support our findings.

The staining characteristics of the lesions studied suggest that these methods will be of use in the interpretation of highly atypical adenomas of the colon in which there is questionable malignancy. A definite blue staining reaction with the PAT/KOH/PAS technique would be strong supportive evidence of malignancy and would indicate the need for additional sectioning or further biopsy material for study. Negative PBT/KOH/PAS would strengthen this interpretation but would not appear to be useful in the presence of equivocal PAT/KOH/PAS results. On the other hand, an entirely normal red reaction would mitigate against malignancy.

Muto et al. (1975) are of the opinion that 'most cancers have been through the polyp-cancer sequence. By this it is meant that invasive adenocarcinoma usually arises in a previous benign tumour ... having the structure of an adenomatous polyp or a villous adenoma'. Furthermore they cite malignancy rates of $2.9 \%, 4 \%$ and $4.8 \%$ for adenomatous polyps, and $55 \%, 40.7 \%, 31.9 \%$, and $20 \%$ for villous adenomas and express the opinion 
that 'we must seek means of identifying which patients with these tumours are at greatest risk from malignant change'. Our results (Table 2) show that villous adenomas with malignant changes all show evidence of blue staining ( 6 appear blue and 7 purple) whereas of the eight benign and atypical, four were red, three purple, and only one blue. It appears, therefore, that our staining technique may be of value for the identification of the onset of malignant change in adenomas. Somewhat surprisingly, in view of the malignancy rates cited above, four of the adenomatous polyps stained purple and only two were red. It must, however, be remembered that all these polyps were from cases in which there was already established adenocarcinoma.

It is a pleasure to acknowledge the superior technical skill of Mrs Odile Polo and Mrs Jana Tomanek, the secretarial assistance of Mrs Elizabeth Barstow, and the generosity of the Canadian Cancer Society of British Columbia, who have defrayed the costs of publishing the colour plates.

\section{References}

Culling, C. F. A. (1974). Handbook of Histopathological and Histochemical Technique, 3rd edition. Butterworth, London.

Culling, C. F. A., Reid, P. E., Burton, J. D., and Dunn, W. L. (1975). A histochemical method of differentiating lower gastrointestinal tract mucin from other mucins in primary or metastatic tumours. Journal of Clinical Pathology, 28, 656-658.

Culling, C. F. A., Reid, P. E., Clay, M. G., and Dunn, W. L. (1974a). The histochemical demonstration of O-acylated sialic acid in gastro-intestinal mucins. Their association with the potassium hydroxide-periodic acid-Schiff effect. Journal of Histochemistry and Cytochemistry, 22, 826-831.

Culling, C. F. A., Reid, P. E., and Dunn, W. L. (1971). The effect of saponification upon certain histochemical reactions of the epithelial mucins of the gastrointestinal tract. Journal of Histochemistry and Cytochemistry, 19, 654-662.

Culling, C. F. A., Reid, P. E., and Dunn, W. L. (1974b). A problem in the removal of sialic acid by acid hydrolysis. Stain Technology, 49, 317-318.

Culling, C. F. A., Reid, P. E., and Dunn, W. L. (1976). A new histochemical method for the identification and visualization of both side chain acylated and nonacylated sialic acids. Journal of Histochemistry and
Cytochemistry, 24, 1225-1230.

Dawson, P. A., and Filipe, M. I. (1976a). An ultrastructural application of silver methenamine to the study of mucin changes in the colonic mucosa adjacent to and remote from carcinoma. Histochemical Journal, 8, 143-158.

Dawson, P. A. and Filipe, M. I.(1976b). An ultrastructural and histochemical study of the mucous membrane adjacent to and remote from carcinoma of the colon. Cancer (Philadelphia), 37, 2388-2398.

Filipe, M. I. (1969). Value of histochemical reactions for mucosubstances in the diagnosis of certain pathological conditions of the colon and rectum. Gut, 10, 577-586.

Filipe, M. I. (1972). The value of a study of the mucosubstances in rectal biopsies from patients with carcinoma of the rectum and lower sigmoid in the diagnosis of premalignant mucosa. Journal of Clinical Pathology, 25, 123-128.

Filipe, M. I., and Branfoot, A. C. (1974). Abnormal patterns of mucous secretion in apparently normal mucosa of large intestine with carcinoma. Cancer (Philadelphia), 34, 282-290.

Filipe, M. I., and Cooke, K. B. (1974). Changes in composition of mucin in the mucosa adjacent to carcinoma of the colon as compared with the normal: a biochemical investigation. Journal of Clinical Patho$\log y, 27,315-318$.

Muto, T., Bussey, H. J. R., and Morson, B. C. (1975) The evolution of cancer of the colon and rectum Cancer (Philadelphia), 36, 2251-2270.

Reid, P. E., Culling, C. F. A., and Dunn, W. L. (1973) Saponification induced increase in the periodic acid Schiff reaction in the gastrointestinal tract. Mechanisn? and distribution of the reactive substance. Journal of Histochemistry and Cytochemistry, 21, 473-482.

Reid, P. E., Culling, C. F. A., and Dunn, W. L. (1974). The histochemical interpretation of the complex results of methylation upon GI tract mucins with special reference to PAS reactivity. Journal of Histochemistry and Cytochemistry, 22, 986-991.

Reid, P. E., Culling, C. F. A., Dunn, W. L., and Clay, M. G. (1976). The use of a transesterification technique to distinguish between certain neuraminidase resistant epithelial mucins. Histochemistry, 46, 203-207.

Reid, P. E., Culling, C. F. A., Dunn, W. L., Ramey, C. W., and Clay, M. G. (1975b). Differences in chemical composition between the epithelial glycoproteins of the upper and lower halves of rat colon. Canadian Journal of Biochemistry, 53, 1328-1332.

Reid, P. E., Culling, C. F. A., Tsang, W. C., Clay, M. G., Ramey, C. W., and Dunn, W. L. (1975a). The demonstration of O-acylated sialic acids in colonic epithelial glycoproteins. Canadian Journal of Biochemistry, 53, 388-391. 